\title{
INFECTIVE ENDOCARDITIS OF THE TRICUSPID VALVE REPORT OF A CASE DUE TO STREPTOCOCCUS VIRIDANS
}

\author{
BY \\ I. M. LIBRACH \\ From the City Isolation Hospital, Nottingham \\ Received September 9, 1946
}

Post-mortem evidence shows that tricuspid lesions are more common than is generally supposed, though they often escape clinical recognition. Gilchrist and Lyon (1933) found that 10 per cent of 109 cases with mitral stenosis had tricuspid stenosis; and Coombs (1924) found that about one-third of all cases of rheumatic endocarditis had tricuspid involvement. That this lesion should pass undiagnosed during life is not surprising, for the physical signs are less constant.

Endocarditis lenta per se is unfortunately a common enough condition, but it affects in the main the left side of the heart. Though a fair number of cases of tricuspid endocarditis due to various organisms, such as gonococcus and pneumococcus, have been reported, comparatively few were due to Strept. viridans. Boldero and Bedford (1924), though they stress the frequency of infective endocarditis and congenital abnormalities, do not mention one case of tricuspid endocarditis in a series of 602 that they collected from various authors, and also confirm the relative infrequency of infective endocarditis of the right heart, mentioning only 30 cases affecting the pulmonary valves, that is 5 per cent. Hentel (1942) mentions one case; Marques et al. (1941) another, a patient with congenital tricuspid stenosis and auricular fibrillation, who developed infective endocarditis as a terminal event; and Hacker (1931) a third with a ruptured spleen and a subcapsular hæmatoma. Melnick et al. (1935) and Oerting (1931) also cite cases - the latter in a male, aged 46, where no blood culture had been done, but the diagnosis was confirmed post-mortem. Horder (1908) in a series of 118 post-mortem examinations, in a total number of 150 cases, mentions 14 with tricuspid involvement, together with mitral and aortic infection or both: no case of tricuspid disease occurred alone and the infection was generally pneumococcal.

\section{CASE RECORD}

A male, aged 50 years, married and a shopkeeper by trade, consulted his doctor three weeks before admission to hospital, with a history of constant cough for seven days, which confined him to bed. On examination then, he had signs of pneumonia, temperature 102, respiration 36 , pulse 120 , but very few physical signs. He complained of pain in the right shoulder and chest midway in front. On exhibition of sulphapyridine, the temperature fell to normal in seven days; the pulse remained at 100 , but rose two days later in spite of further sulphathiazole. The temperature continued to rise to $103^{\circ} \mathrm{F}$., the urine contained albumin, and as the temperature would not settle, he was advised to go into hospital. His previous history and family history were not pertinent.

On admission, $8 / 4 / 43$, he was seen to be a thin frail man of pale colour, temperature 100 , pulse 98, respiration 24. The tongue was a fiery red with some furring. The teeth were very foul. There were no signs in any system, except some impaired resonance over the right basal region with diminished breath sounds and crepitations. The blood pressure was $138 / 90$ and a white blood count was 8850 with 55 per cent neutrophils and 32 per cent 
ymphocytes. A radiogram of the chest showed doubtful unresolved pneumonia in the right upper zone.

He was put on sulphamethazine, $4 \mathrm{~g}$. to start with and $2 \mathrm{~g}$. every six hours. Two days later his temperature had fallen and he felt slightly better. The sulphamethazine was stopped after a total of $26 \mathrm{~g}$. had been given. On 12/4/43, his temperature began to swing upwards again. The chest showed crepitations in both mid-zones, without localizing signs, but it was thought that a lung abscess might be present. On 14/4/43, the total white cells rose to 11,050 of which 89 per cent were neutrophils. Another radiogram of the chest showed doubtful tuberculosis in the right mid-zone, but the sputum contained no acid-fast bacilli. A course of sulphadiazine was started on the $16 \mathrm{th}$, but soon given up as there was no response. There were still no localizing signs but pallor and a polymorphonuclear leucocytosis persisted; no spleen was felt, but a blood culture and Widal were done and both were negative to abortus and the coli-typhoid group. On the 21st the right lung was explored but no pus was found and blood from the lung was sterile on culture.

On the 25th, he was transfused with one pint of blood, and on the 26th with a further pint; he was started again on sulphathiazole, to a total of $32 \mathrm{~g}$., and this seemed to bring the temperature down. Petechiae appeared on the trunk, and on the 30th, systolic and diastolic murmurs were audible at the lower end of the sternum. An infective endocarditis was considered and blood culture which was repeated now grew a pure growth of Strept. viridans. On May 4th, a systolic murmur appeared at the aortic area. On the 7th, the systolic bruit at the base of the sternum was much louder, and suggested tricuspid endocarditis. On the 9th the patient became semi-comatose and died.

Post-mortem examination (Dr. Johns). Generalized petechial rash over body and limbs.

Chest. Right lung, adherent; left, free. Some congestion with early hypostatic pneumonia.

Heart. Gross pericarditis with early pus formation in the pericardial sac. A large mass of rough soft friable vegetations on the posterior cusp of the aortic valve. A smaller, harder, not so friable mass situated on a cusp of the tricuspid valve. No other abnormality of mitral or pulmonary valves. Heart muscle soft and friable. Coronary arteries patent. No gross atheroma. No emboli or thrombosis in great vessels. No sign of chronic rheumatism.

Abdomen. Solid organ congestion. Intestine showed petechial hæmorrhages. No emboli or aneurysm seen.

Brain. Some pus in basal cisterns, which on staining showed streptococci in fair numbers.

Diagnosis. Subacute bacterial endocarditis with pericarditis and terminal meningitis.

\section{Discussion}

This case is interesting from several aspects. The left heart is more frequently injured in endocarditis lenta, except in pneumococcal cases when according to Preble (1904), the relative frequency of right-sided involvement is greater than in acute endocarditis in general. Both sides were involved in this case. Allen (1935) suggests that in cases affecting the right heart, it is due to the fact that the more virulent the organism, the less necessary the violence of the impact necessary to secure its lodgement. He points out that patients with auricular fibrillation rarely develop endocarditis, because blood drools through the valve concerned.

Boldero and Bedford (1924) think that the access of arterial blood to the right heart in congenital cases with patent septa may explain the unusual frequency of dextral endocarditis in this type of case, but in our case both sides were affected, though there was no septal defect. A possible explanation of this is that put forward by Hadfield and Garrod (1942): they postulate that when the pressure of any chamber is raised above the normal, this predisposes to trauma of the related valves and to endocarditis, so that when for example mitral incompetence is present, thus raising the pressure in the pulmonary circuit, right sided involvement is a 
frequent finding. Von Glahn and Pappenheimer (1935) claim that the disease attacks valves, the seat of recent, not old, rheumatic infection, as is usually thought. In this case there was no evidence of any rheumatic infection of a valve, nor was there any rheumatic history, recent or remote. Another well-known important point, is that the Strept. viridans will attack valves that are congenitally malformed, such as bicuspid aortic valves. In this case there were no signs of congenital abnormality.

What is the source of infection in this disease? Horder (1908) showed that infective endocarditis was pre-eminently a streptococcal disease (for streptococci occurred in 66 per cent of his series of 40 cases) and that the types most often found on blood culture were those present in fæces and normal saliva, i.e. $S$. facalis and $S$. salivarius. He also pointed out the absence of any visible focus of infection and later showed that pressure on an apical root abscess forced the contents of the abscess into the lymphatics (quoted by Hadfield and Garrod, 1942). This work has been confirmed by Okell and Elliott (1935), who performed a series of blood cultures on patients undergoing dental extraction, and found bacteræmia in 75 per cent of those examined. In their analysis they agree with Horder that the vast majority of streptococci in infective endocarditis are of the salivary or $S$. viridans type, a fact which has been amply confirmed since. These abound in the mouth and its neighbourhood, especially in the condition of oral sepsis. In 110 cases of pyorrhœa, 12 were found in their series to have a streptococcal bacteræmia at the time of examination, irrespective of operative procedure; i.e. in persons with severely septic mouths, streptococci usually of the viridans type may enter the blood-stream in the absence of any obvious trauma. This leak is obviously not a blood infection and will depend on the rapidity of the phagocytic clearance of the blood-stream, and though of little account in the normal person, may determine an endocarditis in those with diseased or malformed valves. In our case, the man had a mouth full of foul teeth, but unfortunately no culture was made of the organisms therein. The amount of septic absorption taking place must have been on a considerable scale and probably $S$. viridans was present in the gums.

Pus formation is usually not a feature of this disease. It occurred in two places, the pericardium and cerebral meninges both showing $S$. viridans on direct examination-in this case, probably as a terminal event owing to the intense final bacteræmia and pyæmia.

At the time of treating this case, penicillin was not available, and it, therefore, illustrates the uselessness of sulphonamide therapy alone.

A note on the diagnosis of a tricuspid lesion per se, might not be out of place. The characteristic features described are the intense venous engorgement with systolic pulsation of the deep and superficial veins, especially the jugular, due to the large rise in the systolic pressure in the right auricle, and the presystolic and systolic murmurs with reduplication of the second sound at the xiphisternum. Van Bogaert and Mortelmans (1942) emphasize the importance of the persistence of these signs in any case, while Bramwell and King (1942) stress the fact that the $P$ wave of the cardiogram is of unusually high voltage. Finally, in cases with combined mitral and tricuspid lesions, Röslers' sign is valuable, namely, radiography of the heart showing a lack of dilatation of the left auricle due to the tricuspid lesion decongesting the pulmonary circuit, and so relieving the burden of the auricle.

\section{SUMMARY}

A case of endocarditis lenta affecting the aortic and tricuspid valves, in a man of 50 years, is described.

The dangers of oral sepsis in cases with known heart disease are stressed.

In the diagnosis of the individual lesion, the significance of the persistence of systolic and presystolic bruits at the xiphisternum is emphasized.

I would like to thank Dr. Benton, Acting Medical Superintendent, City Hospital, Nottingham, and Dr. Johns, the Hospital Pathologist, for the pathological findings. 


\section{REFERENCES}

Allen, A. C. (1939). Archiv. Path., 27, 399.

Boldero, H. E. A., and Bedford, D. E. (1924). Lancet, 2, 747.

Bramwell, C., and King, J. T. (1942). Principles and Practice of Cardiology, Oxford.

Coombs, C. F. (1924). Rheumatic Heart Disease, Bristol, p. 58.

Gilchrist, A. R. and Lyon, R. M. (1933). Edin. med. J., 40, 587.

Goldburgh, H. L., Baer, S., and Lieber, M. M. (1942). Amer. J. med. Sci., 204, 319.

Hacker, E. (1931). Virchows Arch., 281, 191.

Hadfield, G., and Garrod, L. P. (1942). Recent Advances in Pathology, Churchill, London.

Hentel, W. (1942). Med. Bull. Vet. Admin., 19, 85.

Horder, T. J. (1908). Quart. J. Med., 2, 289.

Marques, A., and Costa, G. (1941). Rev. clin. Sũo Paulo, 9, 187.

Melnick U., and Jaffe, R. H. (1935). Bull. Chicago med. Soc., 37, 550.

Muir, R. (1936). Textbook of Pathology, Amold, London.

Ockerblad, N. F., and Carlson, H. E. (1937). J. Urol., 38, 300.

Oerting, H. (1931). Minnesota Med., 14, 82.

Okell, C. C., and Elliott, S. D. (1935). Lancet, $2,869$.

Pinchin, A. J. S., and Morlock, H. V. (1929). Lancet, 1, 230.

Preble, H. B. (1904). Amer. J. med. Sci., 128, 782.

Van Bogaert, A., and Mortelmans, L. (1942). Rev. Belge Sci. méd., 14, 388.

Von Glahn, W. C., and Pappenheimer, A. M. (1935). Arch. intern. Med., 55, 173.

Wilkinson, J. F. (1929). Lancet, 1, 716. 